extra-pulmonary effects of the disease. In their recently published work [1], it was shown that cigarette smoke condensate can induce mitochondrial dysfunction in an in vitro model. This makes it more tempting to embrace their hypothesis. Nevertheless, one should be cautious in transferring in vitro results to the real situation in patients. We should bear in mind that muscle dysfunction, as one of the so-called systemic effects of COPD, is a complex phenomenon caused by multiple factors, such as oxidative stress, systemic inflammation, muscle disuse and hypoxaemia, among others. Our report on mitochondrial dysfunction at the peripheral muscle level in patients with COPD and low body mass index (and significantly reduced fat-free mass index) [2] should be added to the list of pathological findings at the muscle level in these patients. With regard to the smoking status, there were no differences among the three subpopulations included in our study. None of the subjects smoked at the time of their inclusion in the study. Unfortunately, we do not have data on the accumulative smoking history in this population, which may have helped to shed light on this issue. It should be added that, although causality cannot be established, the significant association between acceptor control ratio and arterial oxygen tension may indicate an abnormal mitochondrial adaptation to long-term repeated episodes of cell hypoxia.

D-J. Slebos and co-workers were also interested in mitochondrial adenosine triphosphate production in our population. Although we did not specifically assess the rate of adenosine triphosphate production, the lower State 3 of respiration in energised mitochondria by succinate in the subpopulation of chronic obstructive pulmonary disease patients with low body mass index reflects a lower oxidative phosphorylation in this subset of patients.

R.A. Rabinovich*, R. Bastos*, E. Ardite*, L. Llinàs*, M. Orozco-Levi ${ }^{\#, \mp}$, J. Gea ${ }^{\#, \text {, J. Vilaró }}{ }^{+}$, J.A. Barberà*, R. Rodriguez-Roisin*, J.C. Fernandez-Checa ${ }^{\S}$ and J. Roca* *Pulmonary Dept (ICT), Hospital Clinic, IDIBAPS, and ${ }^{\S}$ Liver Unit (IMD)-CSIC, IDIBAPS, Universitat de Barcelona, "Muscle and Respiratory System Research Unit, IMIM, CEXS, Universitat Pompeu Fabra, "Respiratory Medicine Dept, Hospital del Mar, and ${ }^{+}$EUIF Blanquerna, Universitat Ramon Llull, Barcelona, Spain.

\section{STATEMENT OF INTEREST}

None declared.

\section{REFERENCES}

1 van der Toorn M, Slebos DJ, de Bruin HG, et al. Cigarette smoke-induced blockade of the mitochondrial respiratory chain switches lung epithelial cell apoptosis into necrosis. Am J Physiol Lung Cell Mol Physiol 2007; 292: L1211-L1218.

2 Rabinovich RA, Bastos R, Ardite E, et al. Mitochondrial dysfunction in COPD patients with low body mass index. Eur Respir J 2007; 29: 643-650.

DOI: $10.1183 / 09031936.00055807$

\title{
Is endobronchial ultrasound additive to routine bronchoscopy in diagnosing sarcoidosis?
}

To the Editors:

In the June issue of the European Respiratory Journal, WoNG et al. [1] describe the utility of endobronchial ultrasound-guided transbronchial needle aspiration (EBUS-TBNA) in a cohort of 65 patients with suspected sarcoidosis. EBUS-TBNA correctly identified noncaseating granuloma in 56 out of 61 patients, who were ultimately diagnosed with sarcoidosis. Thus, the authors' pre-test criteria for sarcoid were correct in 61 (94\%) out of 65 patients. Some readers may therefore argue that EBUS-TBNA was not required if sarcoidosis can be so accurately identified by a combination of clinical and radiographical information. While the authors clearly excluded patients with "suspected or known malignancy", it is nonetheless surprising that no other causes of mediastinal lymphadenopathy, such as lymphoma, metastatic cancer or infection, were identified in this cohort. In fact, in clinical practice, the reason for performing EBUS-TBNA in patients with diffuse mediastinal lymphadenopathy is to rule out malignancy and infection, as it is often difficult to distinguish stage 1 sarcoidosis from, for instance, lymphoma or granulomatous infection. This important study highlights the diagnostic yield of EBUS-TBNA in an inordinately high-prevalence sarcoidosis population but leaves open the question of general applicability

A prospective study is warranted, investigating the added benefit of endobronchial ultrasound-guided transbronchial needle aspiration to routine bronchoscopy with transbronchial lung biopsy in a more heterogeneous population of patients with idiopathic mediastinal lymphadenopathy.

\section{M.P. Kennedy, C.A. Jimenez, R.C. Morice and G.A. Eapen} Dept of Pulmonary Medicine, University of Texas MD Anderson Cancer Center, Houston, TX, USA.

\section{STATEMENT OF INTEREST}

None declared. 


\section{REFERENCES}

1 Wong M, Yasufuku K, Nakajima T, et al. Endobronchial ultrasound: new insight for the diagnosis of sarcoidosis. Eur Respir J 2007; 29: 1182-1186.

\section{DOI: $10.1183 / 09031936.00050907$}

\section{From the authors:}

We would like to thank M.P. Kennedy and co-workers for appropriately highlighting the diversity of differential diagnosis of mediastinal and/or hilar lymphadenopathy. Real-time endobronchial ultrasound-guided transbronchial needle aspiration (EBUS-TBNA) in this category had been shown to be able to detect lymphoma, benign cyst, sarcoidosis and metastatic carcinoma in the thyroid, oesophagus and mesothelioma [1]. In our study [2], patients with suspected or known malignancy, or a previously established diagnosis of sarcoidosis, were excluded. A limitation of the study [2], which was stated in the discussion section, was the high pre-test probability (94\%) of the disease in the study population, which could have led to bias in this high diagnostic yield. Both study centres in our trial are tertiary referral centres for EBUS-TBNA. Patients who were referred to us with clinical and radiological features that suggested sarcoidosis may, therefore, have had other underlying diagnoses already excluded before referral to our centres. This may explain the relatively high pre-test probability. Furthermore, the high pre-test probability is comparable to another large prospective study on the diagnosis of sarcoidosis, in which endoscopic ultrasound-guided fine-needle aspiration was able to identify 50 patients with a final diagnosis of sarcoidosis from the 51 recruited patients with suspected sarcoidosis [3].

M.P. Kennedy and co-workers also suggested that EBUSTBNA is not required if sarcoidosis can be accurately identified by a combination of clinical and radiographical information. However, as discussed in our study [2], pathological specimens are crucial in substantiating a diagnosis of sarcoidosis and excluding other diagnoses, such as tuberculosis,
Hodgkin's lymphoma and malignancy, particularly when systemic steroids are contemplated [4].

Finally, we also feel that a prospective study investigating the added benefit of endobronchial ultrasound-guided transbronchial needle aspiration with transbronchial lung biopsy in a more heterogeneous population of patients with mediastinal lymphadenopathy is needed. The reason for not including transbronchial lung biopsy as part of the comparative procedure in the our study lies in the result of a previous study, which showed that endobronchial ultrasound-guided transbronchial needle aspiration is safe and has a good diagnostic yield, whereas transbronchial lung biopsy has been shown to have risks of pneumothorax and haemoptysis [1].

\section{Wong*,\# and K. Yasufuku*}

*Dept of Thoracic Surgery, Graduate School of Medicine, Chiba University, Chiba, Japan. "Dept of Medicine, The University of Hong Kong, Queen Mary Hospital, Hong Kong SAR, China.

\section{STATEMENT OF INTEREST}

None declared.

\section{REFERENCES}

1 Yasufuku K, Chiyo M, Sekine Y, et al. Real-time endobronchial ultrasound-guided transbronchial needle aspiration of mediastinal and hilar lymph nodes. Chest 2004; 126: 122-128.

2 Wong M, Yasufuku K, Nakajima T, et al. Endobronchial ultrasound: new insight for the diagnosis of sarcoidosis. Eur Respir J 2007; 29: 1182-1186.

3 Annema JT, Veseliç M, Rabe KF. Endoscopic ultrasoundguided fine-needle aspiration for the diagnosis of sarcoidosis. Eur Respir J 2005; 25: 405-409.

4 Newman LS, Rose CS, Maier LA. Sarcoidosis. N Engl J Med 1997; 336: 1224-1234. 